\title{
Optimized Doxycycline-Inducible Gene Expression System for Genetic Programming of Tumor-Targeting Bacteria
}

Dinh-Huy Nguyen, ${ }^{1,2}$ Sung-Hwan You, ${ }^{1}$ An-Trang Ngoc Vo, ${ }^{1,2}$ Hien Thi-Thu Ngo, ${ }^{1,2}$ Khuynh Van Nguyen, ${ }^{1,2}$ Mai Thi-Quynh Duong, ${ }^{1,2}$ Hyon E. Choy, ${ }^{3}$ Miryoung Song, ${ }^{4}$ Yeongjin Hong, ${ }^{3}$ Jung-Joon Min $\mathbb{1}^{1,2,5}$

${ }^{1}$ Institute for Molecular Imaging and Theranostics, Chonnam National University Medical School, Gwangju, 61469, Republic of Korea ${ }^{2}$ Department of Molecular Medicine (BrainKorea21 Plus), Chonnam National University Graduate School, Gwangju, 61469, Republic of Korea

${ }^{3}$ Department of Microbiology, Chonnam National University Medical School, Gwangju, 61469, Republic of Korea

${ }^{4}$ Department of Bioscience and Biotechnology, Hankuk University of Foreign Studies, Yongin, South Korea

${ }^{5}$ Department of Nuclear Medicine, Chonnam National University Medical School, JeonnamGwangju, 61469, Republic of Korea

\begin{abstract}
Purpose: In the programming of tumor-targeting bacteria, various therapeutic or reporter genes are expressed by different gene-triggering strategies. Previously, we engineered pJL87 plasmid with an inducible bacterial drug delivery system that simultaneously co-expressed two genes for therapy and imaging by a bidirectional tet promoter system only in response to the administration of exogenous doxycycline (Doxy). In this multi-cassette expression approach, tetA promoter $\left(\mathrm{P}_{\text {tet } A}\right)$ was 100 -fold higher in expression strength than tetR promoter $\left(\mathrm{P}_{\text {tet }}\right)$. In the present study, we developed pJH18 plasmid with novel Doxy-inducible gene expression system based on a tet promoter.

Procedures: In this system, Tet repressor (TetR) expressed by a weak constitutive promoter binds to tetO operator, resulting in the tight repression of gene expressions by $\mathrm{P}_{\text {tetA }}$ and $\mathrm{P}_{\text {tetR }}$, and Doxy releases TetR from tet $O$ to de-repress $\mathrm{P}_{\text {tet }}$ and $\mathrm{P}_{\text {tetR }}$.

Results: In Salmonella transformed with pJH18, the expression balance of bidirectional tet promoters in pJH18 was remarkably improved $\left(P_{\text {tetA }}: P_{\text {tetR }}=4 \sim 6: 1\right)$ compared with that of pJL87 $\left(P_{\text {teta }}: P_{\text {tetR }}=100: 1\right)$ in the presence of Doxy. Also, the expression level by novel tet system was much higher in Salmonella transformed with pJH18 than in those with pJL87 (80-fold in rluc8 and 5-fold in clyA). Interestingly, pJH18 of the transformed Salmonella was much more stably maintained than pJL87 in antibiotic-free tumor-bearing mice (about 41 -fold), because only pJH18 carries bom sequence with an essential role in preventing the plasmid-free population of programmed Salmonella from undergoing cell division.

Conclusions: Overall, doxycycline-induced co-expression of two proteins at similar expression levels, we exploited bioluminescence reporter proteins with preclinical but no clinical utility. Future validation with clinically compatible reporter systems, for example, suitable for radionuclide imaging, is necessary to develop this system further towards potential clinical application.
\end{abstract}

Correspondence to: Yeongjin Hong; e-mail: yjhong@chonnam.ac.kr,

Jung-Joon Min; e-mail: jjmin@jnu.ac.kr 
Key words: Tet system, Salmonella, Theranostics, Bacteria-mediated gene therapy

\section{Introduction}

The disordered tumor microenvironment is responsible for oxygen deficiency, excessive nutrient leakage, and immune privilege, which can provide a niche in which bacteria such as Clostridium [1], Bifidobacterium [2, 3], Listeria [4, 5], Escherichia coli [6, 7], and Salmonella [8, 9] can survive. Bacteria are well-suited for distinguishing tumor tissue from normal organs because they specifically colonize tumors and proliferate within them $[10,11]$. We previously developed an attenuated strain of Salmonella typhimurium that was defective in guanosine 5 '-diphosphate-3'-diphosphate synthesis ( $\Delta$ ppGpp Salmonella, SAM) and which showed preferential accumulation in tumors, resulting in bacterial numbers that were more than 10,000-fold higher in tumor tissue than in healthy organs [12].

As attenuated bacteria alone are often unable to eradicate malignant tumors, various bacterial species have been subjected to genetic programming to develop tumorselective protein-drug factories [8, 9, 12-15]. Various effector systems have been explored for their ability to express and deliver therapeutic payloads to cancer [11]. Because bacteria tend to localize initially, but transiently, in the liver and spleen [11, 16], various inducible effector systems that utilize external gene triggers such as Larabinose [8, 9, 12, 17], salicylate [18], $\gamma$-irradiation [19], and tetracycline [8] have been actively developed to maximize intratumoral effects while minimizing systemic toxicity.

Tetracycline and its analogues such as doxycycline (Doxy) exhibit several properties of an ideal inducer: they regulate gene expression at very low concentrations (nmol/1 range) and are therefore nontoxic at the necessary levels; they show good bioavailability as they can penetrate both bacterial and animal cells; their stability is suitable for the time courses required for a therapeutic effect; and they are well tolerated in humans, being widely used as antibiotics [8]. The bidirectional tet expression system is simultaneously regulated by the $\mathrm{P}_{\text {tetA }}$ and $\mathrm{P}_{\text {tet } R}$ promoters, which are induced by tetracycline or Doxy $[8,20,21]$. We previously reported a Doxy-inducible bacterial drug delivery system (pJL39) [8] in which a reporter gene and a therapeutic gene under the influence of bidirectional tet promoters were inserted to visualize the targeting process and deliver therapeutic drugs. This inducible system coexpressed dual genes only in response to the administration of Doxy, which was regulated in a dose- and time-dependent manner. Although this inducible system revealed advantages for facilitating controllable therapeutic gene expression in small animal models, the imbalance in promoter strength between $\mathrm{P}_{\text {tetA }}$ and $\mathrm{P}_{\text {tetR }}\left(\mathrm{P}_{\text {tetA }}\right.$ is 100 -fold stronger than $\left.\mathrm{P}_{\text {tet } R}\right)$ is a limiting factor that may hamper achievement of maximal transgene expression levels from the bacteria. The open reading frames (ORFs) of the target gene under $\mathrm{P}_{\text {tet }}$ are located distal to tetR gene, and distal ORFs are often expressed less efficiently than proximal ones; $\mathrm{P}_{\text {tet } R}$ is often relatively less expressed, although there can also be considerable variation according to the bacterial strain and growth conditions.

In the absence of selection pressure, bacteria typically fail to maintain the expression plasmid, particularly in infected animals [22, 23]. Therefore, we employed the bom sequence to ensure stable plasmid maintenance during bacterial growth in an infected mouse model, without the requirement for antibiotic pressure. Bom, a functional sequence for cis mobility, contains nic (known as the origin of transfer, oriT), a site at which a site-specific nick is formed by the relaxation of complex proteins to initiate plasmid mobilization [24, 25]. In fact, depletion of the bom or ori $\mathrm{T}$ sequence resulted in a 95-99\% reduction in the transfer frequency of plasmids in a study of DNA plasmid transfer origin [24].

In this study, we modified the previously reported system pJL39 to promote transcriptional activity of target genes under $\mathrm{P}_{\text {tetR }}$ [8] and developed a novel Doxy-inducible system (pJH18). In the present system, the tet $R$ gene was decoupled from $\mathrm{P}_{\text {tetR }}$ and placed under the control of a weak constitutive promoter $\left(\mathrm{P}_{\text {araB }}\right)$, resulting in direct control of target genes by $\mathrm{P}_{t e t R}$, rather than transcriptional read-through from the tet $R$ transcript, as in pJL39 [8]. Furthermore, the location of bom sequence, which is required for plasmid mobilization [25, 26], plays a critical role in preventing bacterial plasmid loss under normal conditions, resulting in the maintenance of durable protein expression. The present Doxy-inducible system enabled strict regulation and enhanced expression of transgenes in response to a low dose of exogenous Doxy, which achieved the desired biological effect in small animal models.

\section{Results}

\section{Engineering of the Novel Doxy-Inducible Bidirec- tional Expression System}

To improve the expression balance between $\mathrm{P}_{\text {tet } A}$ and $\mathrm{P}_{\text {tet } R}$ and the transfer frequency of the plasmids (Fig. 1), we modified pJL39 to generate pJH18 in which TetR was controlled by the weak constitutive promoter $\mathrm{P}_{\text {araB }}$, and bidirectional tet promoters, $\mathrm{P}_{\text {tetA }}$ and $\mathrm{P}_{\text {tetR }}$, could be used for simultaneous expression of two cargo genes (Fig. 1A). Furthermore, bom sequence was also cloned in this plasmid for its ability to facilitate bacterial maintenance in antibiotic- 
A

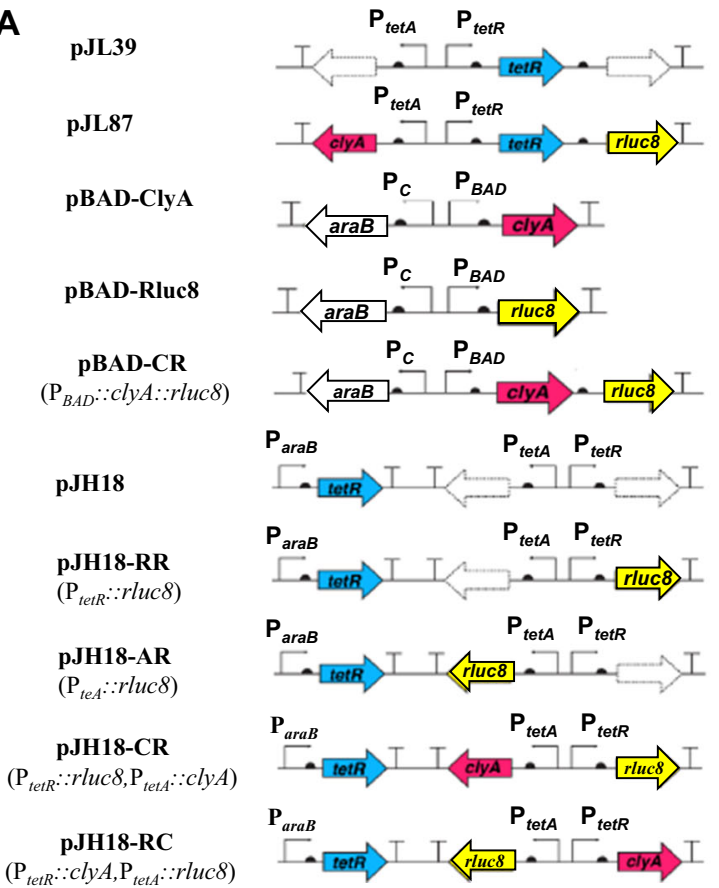

B
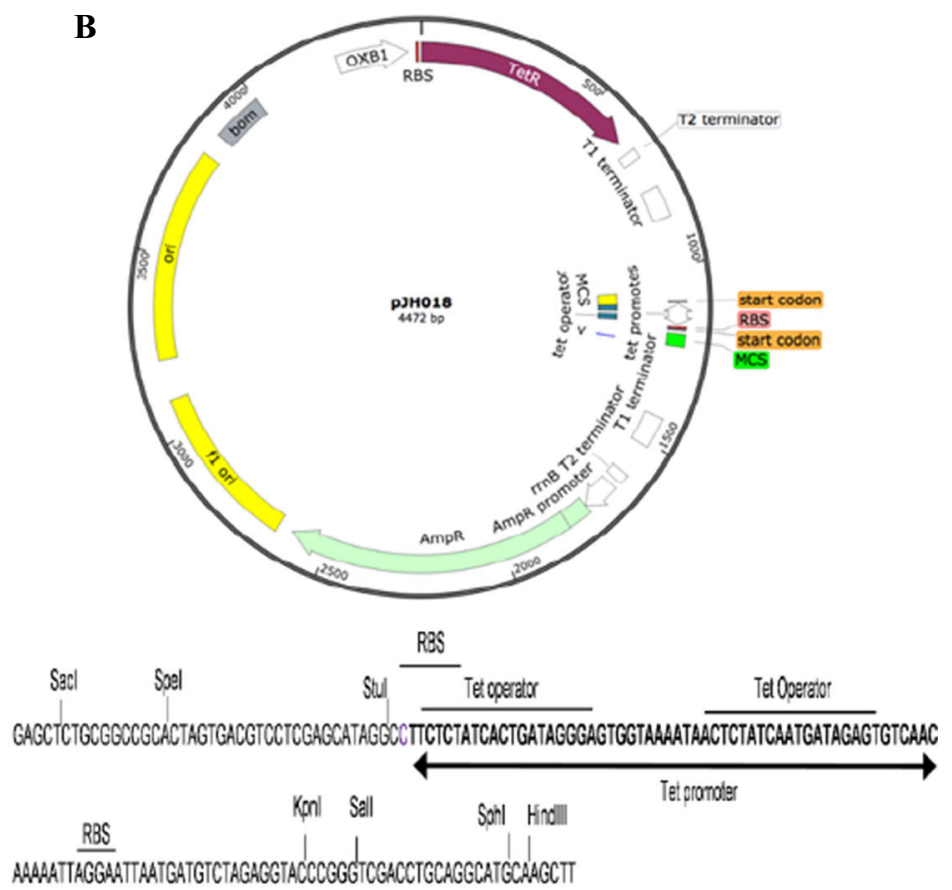

Fig. 1. Schematic diagram of the plasmids used in this study. A Simple description of the plasmids used in this study. Solid big arrows, open reading frames; curved arrows, promoters; T marks, terminators; semicircles, regulator binding sites. B Map and multiple cloning sites (lower part) of the pJH18 vector. A detailed description is provided in the Materials and Methods. MCS, multiple cloning site; RBS, ribosomal binding site; bp, base pairs.

free conditions (Fig. 1B). Using pJH18, we constructed four plasmids in which a reporter gene (rluc8 for Renilla luciferase variant 8$)$ and/or a therapeutic gene (clyA for cytolysin A) were placed under the control of $\mathrm{P}_{\text {tetA }}$ or $\mathrm{P}_{\text {tetR }}$; pJH18-RR encoding rluc8 under $P_{\text {tetR }}$, pJH18-AR encoding rluc8 under $\mathrm{P}_{\text {tet } A}, \mathrm{pJH} 18-\mathrm{CR}$ encoding $c l y A$ and rluc8 under $\mathrm{P}_{\text {tetA }}$ and $\mathrm{P}_{\text {tetR }}$, respectively, and $\mathrm{pJH} 18-\mathrm{RC}$ encoding rluc8 and clyA under $\mathrm{P}_{\text {tetA }}$ and $\mathrm{P}_{\text {tetR }}$, respectively (Fig. 1A).
We transformed $\Delta$ ppGpp Salmonella with the pJH18CR (SAM-CR) or pJL87 (SAM-pJL87) plasmid encoding clyA and rluc8 under $\mathrm{P}_{\text {tetA }}$ and $\mathrm{P}_{\text {tetR }}$, respectively [8]. TetR expression and bom activity were compared between the two transformed bacteria. In SAM-CR, TetR proteins (23 $\mathrm{kDa}$ ) were constitutively expressed in every growth time of Doxy-free condition (Fig. 2A), whereas in SAM-pJL87, TetR was undetectable at any growth time point. We note
A

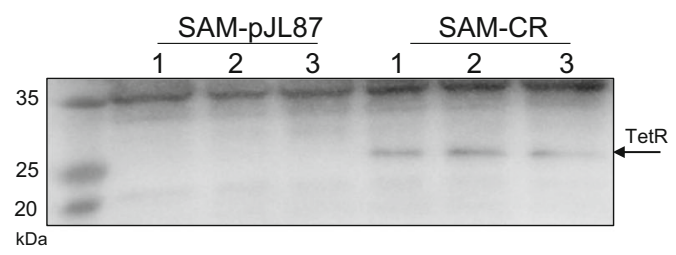

B

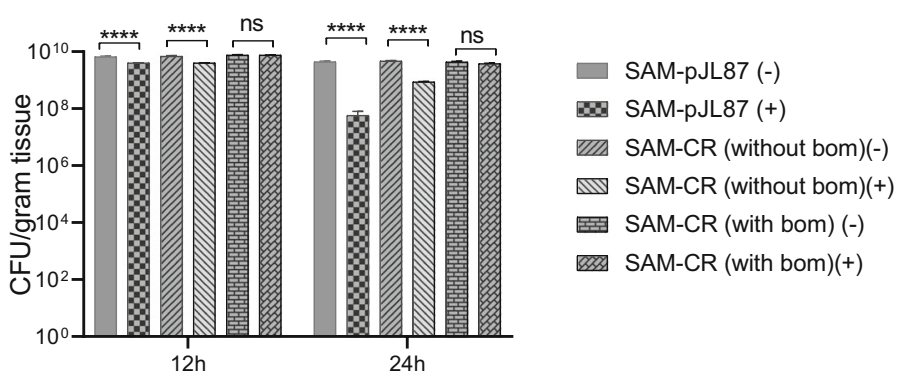

Fig. 2. Measurement of TetR expression and bom gene activity in pJH18 transformants. A Measurement of TetR expression. $\triangle$ ppGpp Salmonella were transformed with pJH18-CR (SAM-CR) or pJLS7 (SAM-pJL87). The transformed bacteria were freshly cultured to exponential (OD600 0.3 and 0.6) and stationary (overnight culture) phases in ampicillin-containing LB broth. Bacterial pellets $\left(8 \times 10^{*} \mathrm{CFU} / \mathrm{ml}\right)$ were dissolved in sample buffer, separated in SDS-PAGE and stained with Coomassie blue (upper panel). TetR proteins ( $25 \mathrm{kDa})$ were detected with western blot using their antibodies (bottom panel). 1, OD600 $0.3 ; 2$, OD600 0.6; 3, overnight culture. B Measurement of bom sequence activity. Bacteria were cultured overnight in the absence of ampicillin, and $10^{-6}$ diluted bacteria were spread on agar plates supplemented with ampicillin and cultured for 1 day. Colonies on each plate were counted, and the total bacterial numbers are depicted after compensation for the dilution factor. + , with ampicillin; -, without ampicillin ( $n=3$ per group. ${ }^{\star \star \star \star} P<0.0001$; ns, not significant, two-way analysis of variance (ANOVA) with Bonferroni's multiple comparison test). All data are shown as mean S.E.M. 
A

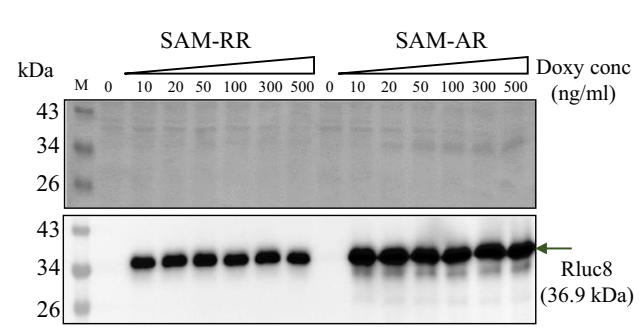

B

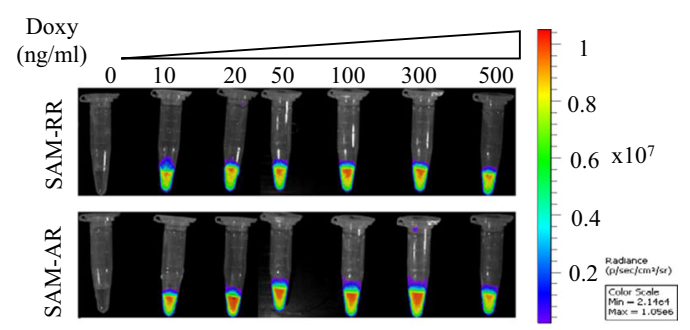

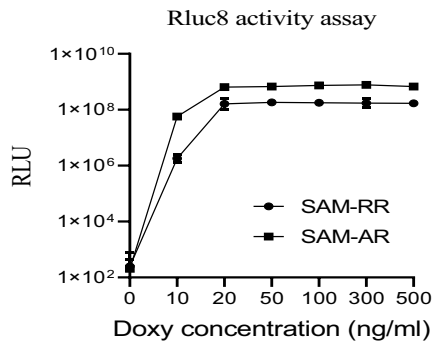

Fig. 3. Comparison of Rluc8 expression by $\mathrm{P}_{\text {tet }}$ and $\mathrm{P}_{\text {tetR. }}$. SAM were transformed with pJH18-RR (SAM-RR) or pJH18-AR (SAM-AR). After culture to OD600 0.5 0.7, the bacteria were added with the indicated concentrations of Doxy and further cultured for $4 \mathrm{~h}$. The same numbers of bacteria $\left(8 \times 10^{8} \mathrm{CFU} / \mathrm{ml}\right)$ were harvested by centrifugation and used for further analysis. A Western blot analysis of Rluc8. After dissolving in sample buffer, the proteins in the bacterial pellets were separated on two SDS-PAGE gels. One gel was stained with Coomassie blue dye (upper panel), whereas the proteins in the other gel were transferred to a nitrocellulose membrane and blotted with anti-Rluc8 antibody. M, marker. B Measurement of luciferase activity. Bacterial pellets were dissolved in PBS with coelenterazine $(1 \mu \mathrm{g} / \mathrm{ml})$. Photographs (right) and light emission rates (left) of each sample were acquired by cooled CCD camera.

that TetR expression relied on Doxy in pJL87, as it is expressed by $\mathrm{P}_{t e t R}[8]$. To assess plasmid maintenance in SAM-pJL87 (without bom) and SAM-CR (with or without bom), these transformants were spread onto agar plates after overnight culture in the absence of ampicillin. After $24 \mathrm{~h}$ of culture, SAM-CR (with bom) showed no significant change of colony numbers in the presence or absence of ampicillin, whereas only $12.9 \%$ of the SAMCR (without bom) and $1.7 \%$ of the SAM-pJL87 colonies remained in the presence of ampicillin compared with those in the absence (Fig. 2B). This result indicates that pJH18 is stably maintained for $24 \mathrm{~h}$, even without antibiotics because it contains bom sequence.

Next, we evaluated Doxy-inducible expression of a reporter gene in cultured SAM transformed with pJH18RR (SAM-RR) or pJH18-AR (SAM-AR). Doxy-induced Rluc8 (36.9 kDa) expression was measured in bacterial cultures after 4-h induction with various Doxy concentrations (Fig. 3). The luciferase activity by Rluc8 was specifically identified only in the presence of Doxy. Doxyinduced Rluc8 expression could be detected as a saturated amount in western blot analysis even at the lowest Doxy concentration $(10 \mathrm{ng} / \mathrm{ml})$ (Fig. 3A). The Rluc8 protein level was 2- to 6-fold higher under $\mathrm{P}_{\text {tetA }}$ than under $\mathrm{P}_{t e t R}$. The western blot result was consistent with that of a luciferase activity assay (Fig. 3B), with luciferase activity being detected even with $10 \mathrm{ng} / \mathrm{ml}$ of Doxy and being saturated at $20 \mathrm{ng} / \mathrm{ml}$ of Doxy. This luciferase activity was 3-fold higher with $\mathrm{P}_{\text {tetA }}$ than with $\mathrm{P}_{\text {tetR }}$ (Fig. 3B).

\section{Enhanced Protein Expression Induced by Doxy in SAM Transformed with pJH18}

Subsequently, we engineered bacteria carrying pJH18-RC (SAM-RC) or pJH18-CR (SAM-CR) and assessed cargo gene expression. In western blot analyses, both Rluc8 (36.9 $\mathrm{kDa})$ and ClyA (34 $\mathrm{kDa})$ proteins could be specifically identified only in the presence of Doxy (Fig. 4A). In SAM$\mathrm{RC}$ and SAM-CR, the expression levels of these proteins were 2- to 6-fold higher under $\mathrm{P}_{\text {teta }}$ than under $\mathrm{P}_{\text {tetR }}$. This result was consistent with Rluc8 enzyme activity assays (Fig. 4B). The ClyA activity secreted from SAM-RC or SAM-CR was also evaluated using a blood agar plate; SAMRC and SAM-CR could lyse blood cells only after Doxy induction (Fig. 4C).

We then compared the cargo expression levels of SAM-CR and SAM-pJL87, which express ClyA and Rluc8 under $\mathrm{P}_{\text {tetA }}$ and $\mathrm{P}_{\text {tetR }}$, respectively (Fig. 5). After Doxy induction, the expression of Rluc8 and ClyA was approximately 80-fold and 5 -fold stronger in SAM-CR than in SAM-pJL87, respectively (Fig. 5A). In particular, Rluc8 expression under the control of $\mathrm{P}_{\text {tetR }}$ showed a remarkable increase in SAM-CR. The expression difference was also reproduced in Rluc8 activity tests. Rluc8 luciferase activity was 30- to 90-fold higher in SAM-CR than in SAM-pJL87, depending on the concentration of Doxy (Fig. 5B). After Doxy induction, SAM-CR showed stronger hemolytic activity than SAM-pJL87 (Fig. 5C). It should be noted that SAM-CR grew well, similar to non-transformed bacteria, despite the strong expression of ClyA and Rluc8 after Doxy induction (Fig. 5D).

The arabinose-inducible expression system using $\mathrm{P}_{B A D}$ promoter has been widely used for precise and strong induction of protein expression in bacteria-based cancer therapy $[9,27]$. Thus, we assessed cargo expression in SAM transformed with pJH18 or pBAD (Fig. S1). The ClyA and Rluc8 expressions driven by $\mathrm{P}_{B A D}$ were comparable to those by $\mathrm{P}_{t e t A}$ and $\mathrm{P}_{t e t R}$. The expression of ClyA and Rluc8 proteins in SAM-pBAD-ClyA was 1.4-fold higher than in SAM-RC $\left(\mathrm{P}_{\text {tetR }}:: c l y A\right)$ but 1.3-fold lower than in SAM-CR $\left(\mathrm{P}_{\text {tet } A}:: c l y A\right)$ (Fig. S1A). This result was consistent with the Rluc8 activity assay (Fig. S1B). Furthermore, we also found that in the single-cassette-polycistronic pBAD system encoding ClyA and Rluc8 (pBAD-CR $\left(\mathrm{P}_{B A D}:: c l y A:: r l u c 8\right)$, Fig. 1A), there was no significant difference in proximal ClyA expression between SAM-pBAD-CR and SAM-CR, but the expression of Rluc8 protein was 3.2-fold 
B

A

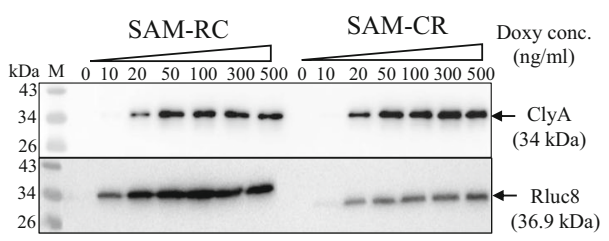

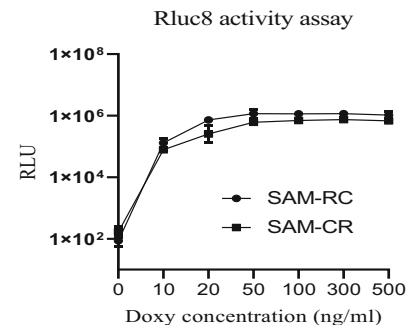

$\mathrm{C}$

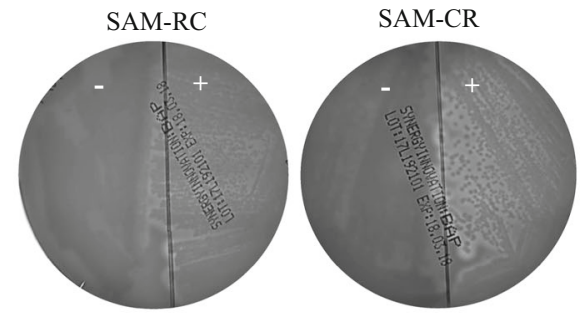

Fig. 4. Expression of ClyA and Rluc8 in pJH18 plasmid. SAM were transformed with pJH18-CR or pJH18-RC. After culture to OD600 0.5 0.7, bacteria were added with the indicated concentration of Doxy and further cultured for $4 \mathrm{~h}$. The same numbers of bacteria $\left(8 \times 10^{8} \mathrm{CFU} / \mathrm{ml}\right)$ were harvested with centrifugation and used for further analysis. A Western blot analysis of ClyA and Rluc8. After dissolving in sample buffer, the bacterial pellets were separated using two SDS-PAGE gels. The proteins were then transferred to nitrocellulose membranes and blotted with anti-ClyA (upper panel) or anti-Rluc8 (bottom panel) antibodies. B Measurement of luciferase activity. Bacterial pellets were dissolved in PBS with coelenterazine (1 $\mu \mathrm{g} / \mathrm{ml})$. Light emission rates of each sample were acquired by cooled CCD camera. $\mathbf{C}$ Hemolysis assay. After culture with Doxy, bacteria were streaked onto a blood agar plate and cultured overnight. +, with Doxy; -, without Doxy.

lower in SAM-pBAD-CR than that in SAM-CR (Fig. S2A). This result was corresponded with the bioluminescence activity (Fig. S2B). The result indicates that the pJH18 system in the present study is appropriate for balanced expression of two genes.

\section{In Vivo Doxy-Inducible Gene expression in Bac- teria of Mouse Tumor Models}

In our previous study, we demonstrated Rluc8 activity driven by $\mathrm{P}_{\text {tetR }}$ in tumor tissues when SAM-pJL87 was injected into mouse tumor models, but its level was 100 -fold lower than that driven by $\mathrm{P}_{\text {tet } A}$ promoter [8]. We evaluated the improvement in the efficiency of gene expression under $\mathrm{P}_{\text {tetR }}$ in SAM-CR by comparing it with that of SAM-pJL87 in CT26 xenograft tumors (Fig. 6). Mice were injected with SAM-CR or SAM-pJL87 via the tail vein and were orally administered Doxy (17 $\mathrm{mg} / \mathrm{kg}$ body weight) at 3 days postinoculum (dpi) (Fig. 6A). Rluc8 activity (driven by $\mathrm{P}_{\text {tetR }}$ ) in CT26 xenografts treated with SAM-CR was 14-fold higher than in those treated with SAM-pJL87 (Fig. 6 B and $\mathrm{C}$ ), and ClyA protein expression (driven by $\mathrm{P}_{\text {tetA }}$ ) was 3 -fold higher in tumor tissues treated with SAM-CR than in those
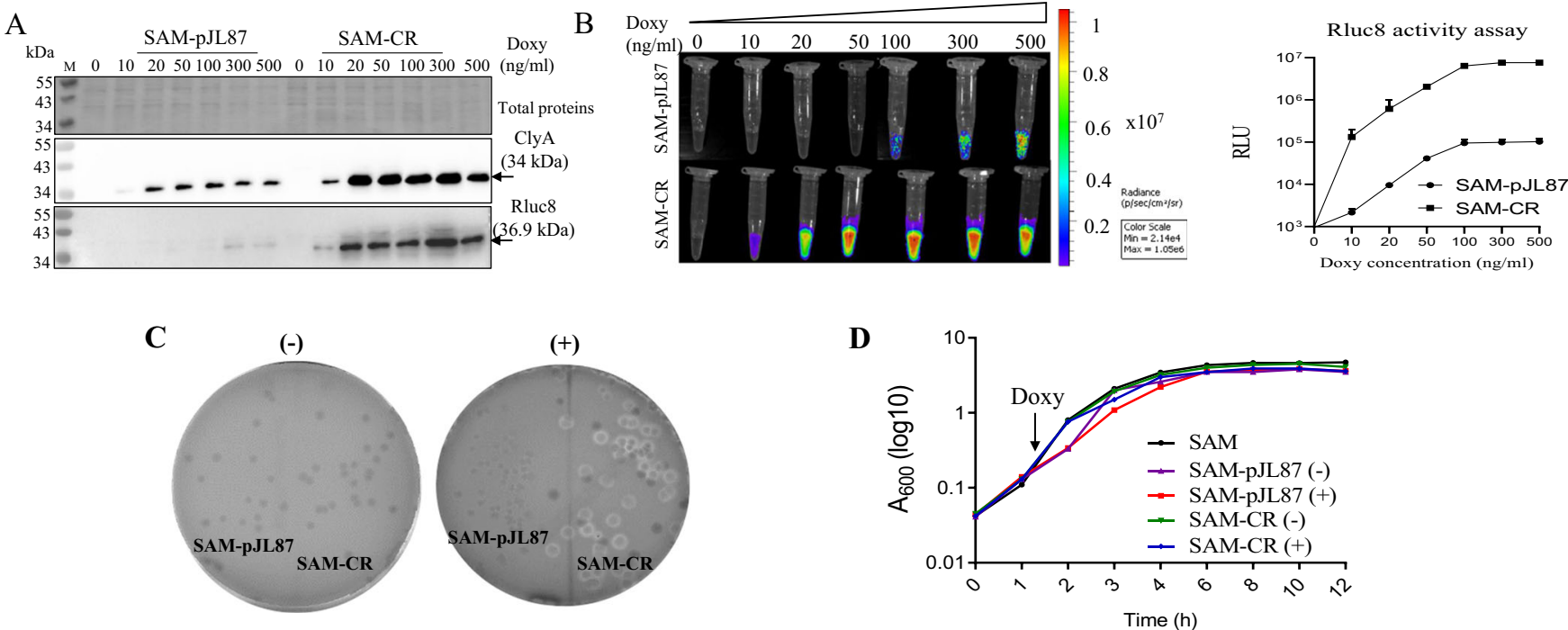

Fig. 5. Comparison of cargo expression in SAM-CR and SAM-pJL87. A Western blot analysis of ClyA and Rluc8 in SAM-CR and SAM-pJL87. B Measurement of luciferase activity in SAM-CR and SAM-pJL87. C Hemolysis assay. Bacteria were streaked onto blood agar plates supplemented with Doxy (300 ng/ml) and cultured overnight. +, with Doxy; -, without Doxy. D Bacterial growth curve. After culture overnight, 100-fold diluted bacteria were inoculated into fresh media and cultured for $1.5 \mathrm{~h}$, after which Doxy $(300 \mathrm{ng} / \mathrm{ml})$ was added and the broth was further cultured. Bacterial growth was measured at OD600 with a spectrophotometer. 
A

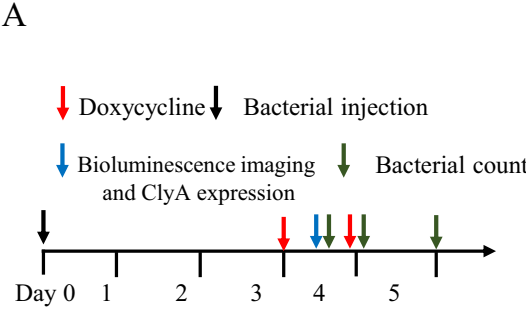

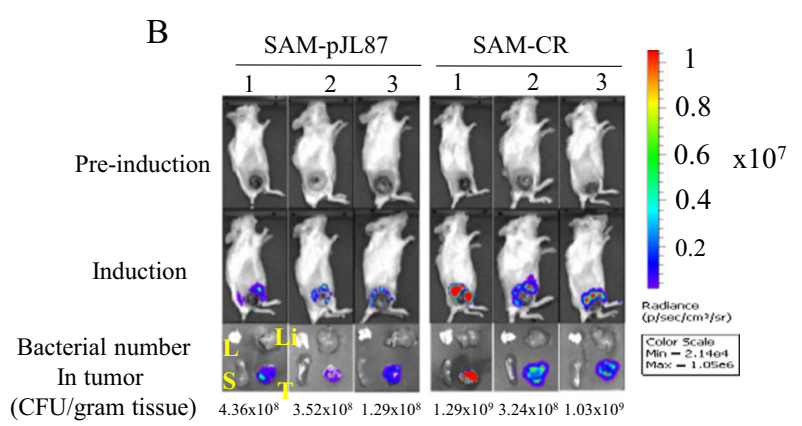

$\mathrm{C}$

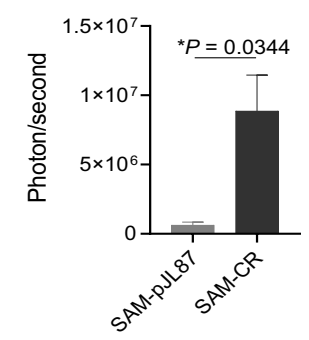

$\mathrm{D}$

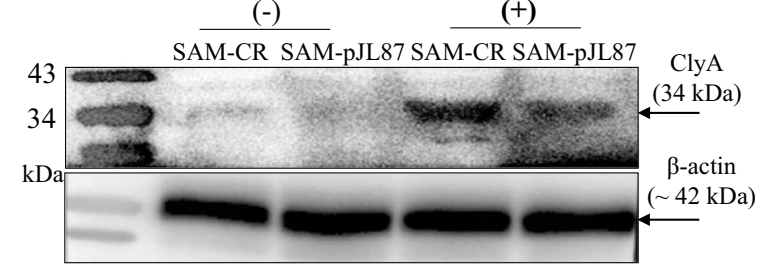

$\mathrm{E}$ SAM-pJL87(-) SAM-CR(-) 88 SAM-pJL87(+) SAM-CR(+)

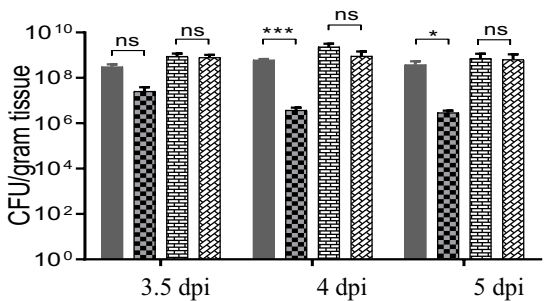

Fig. 6. In vivo cargo expression by SAM-CR and SAM-pJL87 in tumor xenografts. SAM-CR or SAM-pJL87 $(3 \times 107$ CFU/ mouse) were injected via the tail vein of BALB/c mice bearing CT26 (three mice per group). At $3 \mathrm{dpi}$, Doxy (17 mg/kg body weight) was orally administrated. A Experimental schedule. B Bioluminescence imaging of whole body and ex vivo (lung, L; liver, Li; spleen, S; tumor, T). Images were obtained after coelenterazine injection ( $0.7 \mathrm{mg} / \mathrm{kg}$ body weight) via tail vein. C Measurement of luciferase activity in CT26 xenografts. Light emission amounts were acquired by cooled CCD camera. D ClyA identification in tumor tissue by western blot analysis. CT26 tumor tissues were cut out from mice and crushed. The proteins were separated in SDS-PAGE and transferred to nitrocellulose membrane. Western blot was done with anti-ClyA (upper panel) and anti-beta actin (bottom panel) antibodies. +, Doxy administrated mice; -, Doxy non-administrated mice. E Measurement of plasmid maintenance in bacteria. After Doxy administrated in bacteria-injected mice, bacteria were isolated from tumor tissues at the indicated time points and re-cultured in agar plates supplemented with or without ampicillin. One day after culture, colony numbers were counted. +, plates with ampicillin; -, plates without ampicillin $\left(n=3\right.$ per group, $P=0.0123,{ }^{\star \star \star} P=0.0003$; ns, not significant, two-way analysis of variance (ANOVA) with Bonferroni's multiple comparison test). All data are shown as mean + S.E.M.

treated with SAM-pJL87 (Fig. 6D). These results indicate that the novel Doxy-inducible system pJH18 was able to express dual genes in tumor-targeting bacteria much more efficiently than was pJL87.

Interestingly, there were no significant differences in bacterial numbers in the mouse tumors treated with SAMCR until 5 dpi, regardless of Doxy induction (Fig. 6E). By contrast, bacterial numbers in mouse tumors treated with SAM-pJL87 decreased more than 10-fold after Doxy induction compared with numbers before Doxy induction at 3.5, 4, and 5 dpi. We speculate that Doxy induction affects plasmid maintenance in the pJL87 system and that bom sequence in pJH18 is critical to prevent plasmid loss during bacterial growth in tumor tissue.

To further assess the antitumor activity of ClyA expressing bacteria, C57BL/6 mice bearing B16F10 were treated with PBS, attenuated $S$. typhimurium harboring empty vector pJH18 (SAM-E) or pJH18-CR (SAM-CR) through intravenous injection, respectively. Mice revealed a decrease in body weight after bacterial treatment compared with PBS treatment, which might be due to inflammation after bacterial treatment (Fig. S3A). The best therapeutic effect was monitored in mice treated with SAM-CR, and the moderate suppression was observed in mice treated with SAM-E (Fig. S3B and C). These data demonstrated that engineered bacteria secreting ClyA triggered antitumor activity.

We finally evaluated whether in vivo gene expression in the SAM-CR strain could be enhanced by a lower dose of Doxy inducer. When induced with a 10 -fold lower dose of Doxy $(1.7 \mathrm{mg} / \mathrm{kg}$ body weight), bioluminescence was strongly detected in tumors colonized by SAM-CR but not in those colonized by SAM-pJL87 (10-fold higher in the former than in the latter) (Fig. S4A and B). This result indicates that gene expression could be enhanced by transforming bacteria with the novel Doxy-inducible pJH18 system.

\section{Discussion}

This report describes our engineering of a novel Doxyinducible gene expression system (pJH18) for bacteria-based 
cancer therapy in which multiple proteins are expressed in a balanced way by a single vector containing the bidirectional promoters $\mathrm{P}_{\text {tetA }}$ and $\mathrm{P}_{\text {tetR }}$. In particular, decoupling the tetR gene from $\mathrm{P}_{\text {tetR }}$ and placing it under the control of a weak constitutive promoter $\left(\mathrm{P}_{a r a B}\right)$ resulted in promotion of transcriptional activity of target genes under $\mathrm{P}_{\text {tetR }}$. Moreover, the location of a bom sequence in tet expression system, which is required for plasmid mobilization, plays a critical role in preventing bacterial plasmid loss under normal conditions, ensuring maintenance of durable protein expression.

In this study, we demonstrate that the direct regulation of cargo by $\mathrm{P}_{t e t R}$ and the constitutive expression of an appropriate amount of the repressor protein TetR play crucial roles in tightly regulating gene expression in response to Doxy and in maintaining a balance in the expression of target genes by $\mathrm{P}_{t e t A}$ and $\mathrm{P}_{\text {tetR }}$. These results provide a proof-of-principle for a novel Doxy-inducible system (pJH18) to potentiate cargo expression in tumortargeting S. typhimurium. In fact, the engineered Doxyinducible pJH18 system established only around a 3-fold difference in promoter strength between $\mathrm{P}_{\text {tetA }}$ and $\mathrm{P}_{\text {tetR }}$ in comparison with a 100-fold difference with the pJL39 system. In previous systems (pJL39 and pJL87) [8], TetR proteins are controlled directly by $\mathrm{P}_{\text {tetR }}$ promoter bound to tetO operators to block RNA polymerase binding and subsequently inactivate gene transcription by $\mathrm{P}_{\text {tetA }}$ and $\mathrm{P}_{\text {tetR }}$ in the Doxy-free condition (Tet-OFF) $[8,28]$. In the presence of Doxy, the $\mathrm{P}_{\text {tetR }}$ of pJL39 and pJL87 has to initiate constitutive production of TetR repressors, as well as downstream cargo genes, and therefore, a sufficient amount of Doxy $(10 \mathrm{ng} / \mathrm{ml})$ is required to trigger conformational changes in TetR to activate gene expression (Tet-ON) [8]. Furthermore, pJL39 system often caused to express a distal cargo gene such as rluc8 gene much less than a proximal one such as tet $R$ because its operonic structure consisted of a proximal tet $R$ and a distal cargo gene driven by $\mathrm{P}_{t e t R}$ promoter $[8,29,30]$. Thus, we modified pJL39 to generate pJH18, in which $\mathrm{P}_{\text {tetA }}$ and $\mathrm{P}_{\text {tetR }}$ are placed in close proximity to the 5 '-end of genes of interest (GOIs). Such expression system is considered the desired consensus sequence for a higher-affinity RNA polymerase site, leading to improvement of transcriptional activity $[8,30]$.

As recent cancer therapies tend to address approaches using combination therapy [14], a multiplex expression system is desirable in anticancer bacterial engineering to provide diverse therapeutic mechanisms from a single programmed bacterial strain $[8,29,31]$. A single vector system for co-expression of multiple cargos can be achieved by using either multiple expression cassettes or a single expression cassette (polycistronic or monocistronic) [8]. With the multi-cassette approach, differences in the rate of transcription, translation, and stability of RNA and protein products can result in imbalances in the amounts of the protein products. With the single-cassette-polycistronic system, the distal genes/ORFs often show less efficient expression than the proximal genes, and there can also be considerable variation depending on the bacterial strain and the growth conditions $[8,32]$. The present $\mathrm{pJH} 18$ system containing divergent promoters to directly regulate proximal genes should be beneficial for various applications requiring strong release of synergistic multiple genes in a balanced manner. Further study using bacteria that produce multiple anticancer cargos is underway, and the therapeutic effects and mechanisms of action are under evaluation.

A lower Doxy level might be better in gene expression induction, as it could reduce potential side effects, because Doxy is an antibiotic used in human patients with infectious disease [33]. In pJH18 system, low expression of TetR by constitutive weak promoter $\mathrm{P}_{\text {araB }}$ is able to block transcription through binding of TetR to tetO operator in the absence of Doxy. Therefore, much less Doxy (10 20 ng/ml for in vitro use) is required to trigger conformational change in TetR, dissociation from tet $O$, and eventual gene expression. The in vitro result was reproduced by an in vivo study in which bioluminescence of SAM-CR in CT26 xenografts was 10 -fold stronger than that of SAM-pJL87 when induced with a 10 -fold lower dose of Doxy $(1.7 \mathrm{mg} / \mathrm{kg}$ body weight).

Bacteria as an anticancer drug delivery vehicle require durable plasmid inheritance to maintain gene expression through cellular generations. However, plasmid replication and gene expression utilizing the host machinery may result in plasmid extinction in the absence of a selective pressure such as that created by the presence of antibiotics [34]. Plasmid mobilization elements play a major role in the intracellular transfer of bacterial plasmids, and among them, bom sequence is known as a recognition site for the initiation of transfer [26, 35]. In fact, this sequence is necessary for plasmid stability during cell division, and it prolongs durable cargo expression in both in vitro and in vivo applications.

In conclusion, we successfully developed a method for doxycycline-induced co-expression of two proteins at similar expression levels, whereby we exploited bioluminescence reporter proteins with preclinical but no clinical utility. Future validation with clinically compatible reporter systems, for example, suitable for radionuclide imaging, is necessary to develop this system further towards potential clinical application. On the basis of this engineering, we have developed programmed bacterial strains that enable tight regulation of a wide variety of anticancer agents for advanced bacteria-mediated cancer therapy for a broad range of cancer patients.

\section{Materials and Methods}

\section{Bacterial Strains and Cancer Cell Line}

E. coli DH5a-competent cells were purchased from Enzynomics (Daejeon, Korea) and used for all gene cloning work. The SAM bacterium was a $\Delta$ ppGpp-defective S. typhimurium SHJ2037 (relA::cat, spoT::kan) strain that 
is used to measure gene expression and activity in various plasmids [36]. All bacteria were grown in Luria-Bertani (LB) broth at $37{ }^{\circ} \mathrm{C}$ with agitation.

A murine colon carcinoma CT26 cell line was purchased from the American Type Culture Collection (MD, USA). The cells were cultured in high-glucose Dulbecco's Modified Eagle Medium (DMEM) (Welgene, Gyeongsangbukdo, Korea) containing $10 \%$ fetal bovine serum (FBS) and 1 $\%$ penicillin-streptomycin and were incubated at $37^{\circ} \mathrm{C}$ in an incubator with $5 \% \mathrm{CO}_{2}$.

\section{Plasmid Construction of a Divergent Gene Expression System Controlled by Doxy}

$\mathrm{pJH} 18$, a plasmid with novel bidirectional tet promoter $\left(\mathrm{P}_{\text {tetA }}\right.$ and $\mathrm{P}_{\text {tetR }}$ ), was engineered as follows. Using pJL39 plasmid as a template $[8,37]$, the tetR OFR [37] was amplified by polymerase chain reaction (PCR) with a TetR-R(EcoRI) and TetR-F(XbaI) primer set. After digestion with EcoRI and $X b a \mathrm{I}$, the fragment was introduced into the same enzyme sites of pBAD plasmid and named pBAD-TetR [9]. The plasmid has tetR ORF driven by $\mathrm{P}_{B A D}$ promoter. The fragment containing a bidirectional tet promoter and multiple cloning sites at both ends (MCSI and MCSII) was amplified by PCR using a Tet-F(AflII) and Tet-R(HindIII) primer set with pJL39 as a template. The amplified fragment was digested with AflII and HindIII and inserted into the same enzyme sites of pBAD-TetR, which was called pTetBAD. The bom fragment was amplified by the bom-F(PciI) and bom-R(NheI) primer set using pBAD-Rluc8 as a template and was cloned between PciI and NheI enzyme sites of pTet-BAD after enzyme digestion. It was named pTetII which has lost $\mathrm{P}_{B A D}$ promoter. Finally, a constitutive promoter $\mathrm{P}_{a r a B}$ was amplified by a $\mathrm{P}_{a r a B^{-}} \mathrm{F}(\mathrm{MfeI})$ and $\mathrm{P}_{a r a B^{-}}$ $\mathrm{R}(\mathrm{MfeI})$ primer set using E. coli DH5 $\alpha$ genomic DNA as a template and was cloned upstream of tetR ORF in pTetII using the Gibson assembly method (New England Biolabs, MA, USA). The resulting plasmid was named pJH18 and used it as a backbone in this study.

Various cargo gene fragments were amplified by PCR. The rluc8 gene was driven by $\mathrm{P}_{\text {tetA }}$ promoter $\left(\mathrm{P}_{\text {tet } A}:\right.$ :rluc 8$)$ and was amplified by an Rluc8/ClyA-P $\mathrm{P}_{\text {tet } A}-\mathrm{F}$ (StuI) and Rluc8-P tetA $-\mathrm{R}(\mathrm{SpeI})$ primer set using the pBAD-Rluc8 plasmid as a template. The rluc 8 gene driven by $\mathrm{P}_{t e t R}$ promoter $\left(\mathrm{P}_{t e t R}::\right.$ rluc8) was amplified by an Rluc8- $\mathrm{P}_{t e t R^{-}}$ $\mathrm{F}(\mathrm{KpnI})$ and Rluc8- $\mathrm{P}_{\text {tet }} \mathrm{R}$ (HindIII) primer set using the pBAD-Rluc8 plasmid as a template.

The clyA gene driven by $\mathrm{P}_{\text {tetA }}$ promoter $\left(\mathrm{P}_{\text {tet } A}:: c l y A\right)$ was amplified by an Rluc8/ClyA-P tet $^{-} \mathrm{F}$ (StuI) and ClyA-P tet $^{-}$ $\mathrm{R}(\mathrm{SacI})$ primer set using the pBAD-ClyA plasmid as a template. The $c l y A$ driven by $\mathrm{P}_{\text {tetR }}$ promoter $\left(\mathrm{P}_{\text {tetR }}: \because c l y A\right)$ was amplified by a ClyA-P $\mathrm{P}_{\text {tet }}-\mathrm{F}(\mathrm{KpnI})$ and ClyA- $\mathrm{P}_{\text {tet }}-\mathrm{R}$ (SalI) primer set using the pBAD-ClyA plasmid as a template.

The PCR fragments were digested by the indicated restriction enzymes and cloned into the corresponding sites in pJH18. The resulting plasmids were named pJH18-RR $\left(\mathrm{P}_{\text {tetR }}:\right.$ :rluc8), pJH18-AR ( $\mathrm{P}_{\text {tet } A}:$ :rluc 8), pJH18-CR $\left(\mathrm{P}_{\text {tet } A}:: c l y A, \mathrm{P}_{\text {tetR }}::\right.$ rluc8), and $\mathrm{pJH} 18-\mathrm{RC}\left(\mathrm{P}_{\text {tet } A}::\right.$ rluc8, $\left.\mathrm{P}_{\text {tet } A}:: \operatorname{cly} A\right)$.

To evaluate the expression level of distal genes/ORFs, we developed pBAD-ClyA-Rluc8 from pBAD-ClyA system. The $r l u c 8$ gene was amplified by an Rluc8- $\mathrm{P}_{B A D}-\mathrm{F}$ (SalI) and Rluc8- $\mathrm{P}_{B A D}-\mathrm{F}(\mathrm{SphI})$ primer set using the pBAD-Rluc8 plasmid as template. The PCR products were digested by the indicated restriction enzymes and cloned into the corresponding sites in pBAD-ClyA for generating pBADClyA-Rluc8 $\left(\mathrm{P}_{B A D:: c l y A:: c l u c 8}\right)$.

The primers and plasmids used in cloning were shown in Tables S1 and S2 and Fig. 1.

\section{Western Blot Analysis and Luciferase Activity Assay}

For western blot analysis, SAM bacteria transformed with the plasmids were incubated overnight in LB medium containing ampicillin $(100 \mu \mathrm{g} / \mathrm{ml})$. The cultured bacteria were inoculated into a medium with 1:100 dilutions and cultured. At an optical density of 0.6 at $600 \mathrm{~nm}\left(\mathrm{OD}_{600}\right)$, Doxy $[0-500 \mathrm{ng} / \mathrm{ml}$ in ethanol (w/v)] was added, and the bacteria were further cultured. After $4 \mathrm{~h}$, the bacteria were precipitated with centrifugation at $4000 \mathrm{rpm}$ for $5 \mathrm{~min}$. After simple washing with phosphate-buffered saline (PBS), the bacterial pellets were dissolved in sodium dodecyl sulfate (SDS) buffer containing $0.2 \% \beta$ mercaptoethanol (Sigma-Aldrich, Darmstadt, Germany) and were heat-treated for $5 \mathrm{~min}$ at $95{ }^{\circ} \mathrm{C}$. The dissolved bacterial proteins $\left(0.5 \mathrm{OD}_{600}\right.$ equivalents per lane) were separated in $15 \%$ SDS-PAGE (40\% Acrylamide, Tris$\mathrm{HCl}$ (pH 6.8)/Tris base ( $\mathrm{pH}$ 8.8), SDS, ammonium persulfate (APS), TEDMED) in running buffer (Tris base, glycine, sodium dodecyl sulfate) and then transferred to nitrocellulose membrane (GE Healthcare, MA, USA) by transfer buffer (Tris base, glycine). The membranes were incubated in blocking buffer $[5 \%(\mathrm{w} / \mathrm{v})$ skim milk in Trisbuffered saline buffer (Tris base, $\mathrm{NaCl}$ ) containing $0.1 \%$ Tween 20 (Sigma-Aldrich, Darmstadt, Germany) (TBS-T)] at room temperature for $1 \mathrm{~h}$. After decanting the blocking buffer (TBS $+5 \% \mathrm{w} / \mathrm{v}$ skim milk), primary antibodies in $1 \mathrm{X}$ TBS with $1 \%$ skim milk were added to the membranes, and they were incubated for $2 \mathrm{~h}$. After decanting the primary antibodies and simple washing with TBS-T (500 ml TBS $1 \mathrm{X}+500 \mu \mathrm{l}$ Tween) (three times), secondary antibodies conjugated with horseradish peroxidase (HRP) were added to the membranes and incubated for $1 \mathrm{~h}$. Before adding the chemiluminescence HRP substrate (Merck Millipore, MA, USA), the membranes were washed with TBS-T three times. The specific protein bands against each antibody were detected with a ChemiDoc ${ }^{\mathrm{TM}}$ XRS + system imager (BIO-RAD, California, 
USA). All antibodies used in this study are listed in Table S3.

For the luciferase activity assay, SAM bacteria transformed with the $\mathrm{pJH} 18$ plasmids containing the Rluc 8 gene were induced by increasing the concentration of Doxy in the early exponential phase $\left(\mathrm{OD}_{600}, 0.5 \sim 0.7\right)$ for $4 \mathrm{~h}$. The bacterial pellets $\left(\mathrm{OD}_{600}, 0.5\right.$ per sample) were resuspended in $100 \mu \mathrm{l}$ of PBS to measure Rluc8 activity immediately after addition of $5 \mu \mathrm{l}$ of coelenterazine $(0.2 \mu \mathrm{g} / \mu \mathrm{l})$ at room temperature. Light emission was measured using a Microlumat Plus LB96V luminometer (Berthold Technologies, Bad Wildbad, Germany) or a Lumina S5 in vivo imaging system (IVIS; Perkin Elmer, MA, USA) with an exposure every $1 \mathrm{~s}$. The measured values were calculated as relative luminescence units [38].

\section{Measurement of Plasmid Loss During Bacterial Culture}

SAM bacteria transformed with the indicated plasmids (pBAD-clyA, pJH18-CR (without bom sequence), pJL87, pJH18-CR) were first grown overnight on LB agar plates containing ampicillin. Single colonies from plates were inoculated into $5-\mathrm{ml} \mathrm{LB}$ broth without ampicillin at $37{ }^{\circ} \mathrm{C}$ with shaking. After 1 day, the bacteria were serially 10 fold diluted with LB broth, and $100 \mu \mathrm{l}$ of the diluted bacteria were spread out on a plate with or without ampicillin and incubated overnight at $37{ }^{\circ} \mathrm{C}$. The numbers of colonies in each plate were counted the next day.

\section{Animal Grouping and Housing, Mouse Tumor Model, and Bacterial Injection}

Female 6-week-old BALB/c mice and C57BL/6 ( 20 g in body weight) were purchased from Orient (Seongnam, Korea) and keep in specific pathogen-free conditions of mouse room for 1 week prior to starting any experiments. The mice were housed in a group of 3-5 mice in per plexiglass cage $(22.3 \times 26.8 \times 13.0 \mathrm{~cm})$ under $12 \mathrm{~h} / 12 \mathrm{~h}$ at light/dark cycle (light on at 7:00 am) in a temperaturecontrolled room $\left(22-24{ }^{\circ} \mathrm{C}\right)$ and $40-60 \%$ humidity, with free access to tap water and standard chow diet (PMI Nutrition International, LLC 4001 Lexington Avenue North, Arden Hills, MN 55126). Cages were lined with full autoclave wood fiber (Northeastern Product Corp., Warrensburg, NY, 12885) and replaced by clean cages 2 times/week. No enrichment material was used inside the cage. At the moment of the experiments, mice were weighing approximately $18 \mathrm{~g}$ and aging 7 weeks. The animals were randomly selected from different cages to house them in one new cage for tumor implantation, and each mouse was used for experiment only once. All experimental processes were performed from 11:00 to 7:00 pm. After culture in DMEM media, CT26 cells $\left(10^{6}\right.$ cells per mouse) or $\mathrm{B} 16 \mathrm{~F} 10$ cells $\left(5 \times 10^{5}\right.$ cells per mouse) were detached and subcutaneously injected into mice anesthetized with $2 \%$ isoflurane. After cell injection, it took about 12 days for the tumor volume to reach $150-180 \mathrm{~mm}^{3}$. All animal care and experimental procedures were performed following the guidelines of the Animal Care and Use Committee, Chonnam National University (Gwangju, Korea), the National Centre of the Replacement, Refinement and Reduction on Animals in Research [39].

For bacterial injections, SAM transformants cultured overnight were inoculated into fresh LB media containing ampicillin in a 100-fold dilution ratio and were further incubated until an $\mathrm{OD}_{600}$ of 2 2.5 (early stationary phase) was attained. After centrifugation at $4000 \mathrm{rpm}$ for $5 \mathrm{~min}$, bacterial pellets were washed with PBS twice. Bacteria (3 $\times 10^{7} \mathrm{CFU} / 100 \mu \mathrm{l}$ in PBS) were intravenously injected into the mice via the tail vein. To induce cargo genes in SAM transformants, the indicated amounts of Doxy (SigmaAldrich, MO, USA) were orally administered using gavage (starting at 3-day postinoculum, once a day after $1 \mathrm{~h}$ of fasting) [8]. Tumor volume $\left(\mathrm{mm}^{3}\right)$ was calculated using the formula (length $\times$ height $\times$ width) $/ 2$ of the tumor in millimeters [8]. Most data analysis and experiments were blinded to prevent any bias.

\section{In Vivo Bioluminescence Imaging and Detection of the Cargo Molecule in Tumor Tissues}

To obtain bioluminescence images $12 \mathrm{~h}$ after oral Doxy administration, tumor-bearing mice were anesthetized with 2 $\%$ isoflurane and then received coelenterazine $(0.7 \mathrm{mg} / \mathrm{kg}$ body weight) through intravenous injection. After $1 \mathrm{~min}$, bioluminescence imaging was acquired from the mice using an IVIS system, as described previously [17, 40].

To measure the existence of cargo molecules, tumor tissues were obtained from mice and homogenized in protein extraction solution $(20 \mathrm{mM}$ Tris- $\mathrm{Cl} \mathrm{pH} 6.8,150 \mathrm{mM} \mathrm{NaCl}$, $5 \mathrm{mM}$ EDTA, $1 \%$ NP-40, protease inhibitor $1 \mathrm{X}$ (Xpert protease inhibitor cocktail solution 100X, GenDEPOT)). The homogenized tissue samples were centrifuged and filtered through a $0.2-\mu \mathrm{m}$ filter. A total of $20 \mu \mathrm{g}$ of proteins were separated using $15 \%$ SDS-PAGE, and western blot analysis was performed with specific antibodies.

\section{Bacterial Counting in Tumor Tissues}

After sacrificing the mice, tumors and other organs were imaged with IVIS. Tumors were homogenized and filtered as the sample preparation for the western blot analysis. The filtrates were serially diluted with PBS and spread out on LB agar plates with or without ampicillin. After overnight culture, the bacteria were enumerated, and the results were expressed as CFU/g of organ. 


\section{Statistical Analysis}

Statistical analysis was performed using GraphPad Prism 8.0 software with $p<0.05$ considered significant. Two independent groups were compared using unpaired twotailed $t$-tests. Comparison of multiple experimental groups was evaluated using one- or two-way ANOVA with multiple comparisons post hoc test to obtain multiplicity-adjusted $P$ value. All data are shown as mean $\pm \mathrm{SEM}$.

Supplementary Information. The online version contains supplementary material available at https://doi.org/10.1007/s11307-021-01624-x.

Funding. This research was supported by the National Research Foundation of Korea (NRF) grants NRF-2017R1A2B3012157 and NRF2018R1A5A2024181. Y. H. was supported by the NRF of Korea grant, funded by the MSIT (Nos. 2018R1A5A2024181 and NRF2020M3A9G3080282).

\section{Declarations}

\section{Conflict of Interest}

The authors declare that they have no conflict of interest.

Open Access This article is licensed under a Creative Commons Attribution 4.0 International License, which permits use, sharing, adaptation, distribution and reproduction in any medium or format, as long as you give appropriate credit to the original author(s) and the source, provide a link to the Creative Commons licence, and indicate if changes were made. The images or other third party material in this article are included in the article's Creative Commons licence, unless indicated otherwise in a credit line to the material. If material is not included in the article's Creative Commons licence and your intended use is not permitted by statutory regulation or exceeds the permitted use, you will need to obtain permission directly from the copyright holder. To view a copy of this licence, visit http:// creativecommons.org/licenses/by/4.0/.

\section{References}

1. Janku F, Zhang HH, Pezeshki A, Goel S, Murthy R, Wang-Gillam A, Shepard DR, Helgason T, Masters T, Hong DS, Piha-Paul SA, Karp DD, Klang M, Huang SY, Sakamuri D, Raina A, Torrisi J, Solomon SB, Weissfeld A, Trevino E, DeCrescenzo G, Collins A, Miller M, Salstrom JL, Korn RL, Zhang L, Saha S, Leontovich AA, Tung D, Kreider B, Varterasian M, Khazaie K, Gounder MM (2021) Intratumoral injection of Clostridium novyi-NT spores in patients with treatment-refractory advanced solid tumors. Clin Cancer Res 27(1):96-106

2. Cronin M, Morrissey D, Rajendran S, el Mashad SM, van Sinderen D, O'Sullivan GC, Tangney M (2010) Orally administered bifidobacteria as vehicles for delivery of agents to systemic tumors. Mol Ther 18(7):1397-1407

3. Longhi G, Van Sinderen D, Ventura M, Turroni F (2020) Microbiota and cancer: the emerging beneficial role of bifidobacteria in cancer immunotherapy. Front Microbiol 11:2188

4. Vitiello M, Evangelista M, di Lascio N, Kusmic C, Massa A, Orso F, Sarti S, Marranci A, Rodzik K, Germelli L, Chandra D, Salvetti A, Pucci A, Taverna D, Faita F, Gravekamp C, Poliseno L (2019) Antitumoral effects of attenuated Listeria monocytogenes in a genetically engineered mouse model of melanoma. Oncogene 38(19):3756-3762

5. Yang Y, Hou J, Lin Z, Zhuo H, Chen D, Zhang X, Chen Y, Sun B (2014) Attenuated Listeria monocytogenes as a cancer vaccine vector for the delivery of CD24, a biomarker for hepatic cancer stem cells. Cell Mol Immunol 11(2):184-196

6. Kang S-R, Jo EJ, Nguyen VH, Zhang Y, Yoon HS, Pyo A, Kim DY, Hong Y, Bom HS, Min JJ (2020) Imaging of tumor colonization by Escherichia coli using 18F-FDS PET. Theranostics 10(11):4958-4966

7. Jiang S-N, Phan TX, Nam TK, Nguyen VH, Kim HS, Bom HS, Choy HE, Hong Y, Min JJ (2010) Inhibition of tumor growth and metastasis by a combination of Escherichia coli-mediated cytolytic therapy and radiotherapy. Mol Ther 18(3):635-642

8. Jiang SN, Park SH, Lee HJ, Zheng JH, Kim HS, Bom HS, Hong Y, Szardenings M, Shin MG, Kim SC, Ntziachristos V, Choy HE, Min JJ (2013) Engineering of bacteria for the visualization of targeted delivery of a cytolytic anticancer agent. Mol Ther 21(11):1985-1995

9. Nguyen VH, Kim HS, Ha JM, Hong Y, Choy HE, Min JJ (2010) Genetically engineered Salmonella typhimurium as an imageable therapeutic probe for cancer. Cancer Res 70(1):18-23

10. Forbes NS (2010) Engineering the perfect (bacterial) cancer therapy. Nat Rev Cancer 10(11):785-794

11. Zhou S, Gravekamp C, Bermudes D, Liu K (2018) Tumour-targeting bacteria engineered to fight cancer. Nat Rev Cancer 18(12):727-743

12. Zheng JH, Nguyen VH, Jiang SN, Park SH, Tan W, Hong SH, Shin MG, Chung IJ, Hong Y, Bom HS, Choy HE, Lee SE, Rhee JH, Min JJ (2017) Two-step enhanced cancer immunotherapy with engineered Salmonella typhimurium secreting heterologous flagellin. Sci Transl Med 9(376):eaak9537

13. Chowdhury S, Castro S, Coker C, Hinchliffe TE, Arpaia N, Danino T (2019) Programmable bacteria induce durable tumor regression and systemic antitumor immunity. Nat Med 25(7):1057-1063

14. Gurbatri CR, Lia I, Vincent R, Coker C, Castro S, Treuting PM, Hinchliffe TE, Arpaia N, Danino T (2020) Engineered probiotics for local tumor delivery of checkpoint blockade nanobodies. Sci Transl Med 12(530):eaax0876

15. Leventhal DS, Sokolovska A, Li N, Plescia C, Kolodziej SA, Gallant CW, Christmas R, Gao JR, James MJ, Abin-Fuentes A, Momin M, Bergeron C, Fisher A, Miller PF, West KA, Lora JM (2020) Immunotherapy with engineered bacteria by targeting the STING pathway for anti-tumor immunity. Nat Commun 11(1):1-15 2739

16. Duong MT-Q, Qin Y, You S-H, Min J-J (2019) Bacteria-cancer interactions: bacteria-based cancer therapy. Exp Mol Med 51(12):115

17. Le UN et al (2011) Engineering and visualization of bacteria for targeting infarcted myocardium. Mol Ther 19(5):951-959

18. Royo JL, Becker PD, Camacho EM, Cebolla A, Link C, Santero E, Guzmán CA (2007) In vivo gene regulation in Salmonella spp. by a salicylate-dependent control circuit. Nat Methods 4(11):937-942

19. Ganai S, Arenas R, Forbes N (2009) Tumour-targeted delivery of TRAIL using Salmonella typhimurium enhances breast cancer survival in mice. Br J Cancer 101(10):1683-1691

20. Williams KJ, Joyce G, Robertson BD (2010) Improved mycobacterial tetracycline inducible vectors. Plasmid 64(2):69-73

21. Zabala M, Wang L, Hernandez-Alcoceba R, Hillen W, Qian C, Prieto J, Kramer MG (2004) Optimization of the Tet-on system to regulate interleukin 12 expression in the liver for the treatment of hepatic tumors. Cancer Res 64(8):2799-2804

22. Krute CN, Krausz KL, Markiewicz MA, Joyner JA, Pokhrel S, Hall PR, Bose JL (2016) Generation of a stable plasmid for in vitro and in vivo studies of Staphylococcus species. Appl Environ Microbiol 82(23):6859-6869

23. Inoue Y (1997) Spontaneous loss of antibiotic-resistant plasmids transferred to Escherichia coli in experimental chronic bladder infection. Int J Urol 4(3):285-288

24. Guiney DG, Yakobson E (1983) Location and nucleotide sequence of the transfer origin of the broad host range plasmid RK2. Proc Natl Acad Sci 80(12):3595-3598

25. Finnegan J, Sherratt D (1982) Plasmid ColE1 conjugal mobility: the nature of bom, a region required in cis for transfer. Mol Gen Genet MGG 185(2):344-351

26. Roessler E, Fenwick R, Chinault A (1985) Analysis of mobilization elements in plasmids from Shigella flexneri. J Bacteriol 161(3):12331235

27. Ha JM, Vu NH, Min JJ, Bom HS (2009) Characterization of Larabinose -induced gene expression in Salmonella typhimurium. J Nucl Med:50 
28. Yamada M, Suzuki Y, Nagasaki SC, Okuno H, Imayoshi I (2018) Light control of the Tet gene expression system in mammalian cells. Cell Rep 25(2):487-500 e486

29. Kerrigan JJ, Xie Q, Ames RS, Lu Q (2011) Production of protein complexes via co-expression. Protein Expr Purif 75(1):1-14

30. Kim KJ, Kim HE, Lee KH, Han W, Yi MJ, Jeong J, Oh BH (2004) Two-promoter vector is highly efficient for overproduction of protein complexes. Protein Sci 13(6):1698-1703

31. Tan S (2001) A modular polycistronic expression system for overexpressing protein complexes in Escherichia coli. Protein Expr Purif 21(1):224-234

32. Schuetze T, Meyer V (2017) Polycistronic gene expression in Aspergillus niger. Microb Cell Factories 16(1):162

33. Das TA, Tenenbaum L, Berkhout B (2016) Tet-on systems for doxycycline-inducible gene expression. Current gene therapy 16(3):156-167

34. Wein T, Hülter NF, Mizrahi I, Dagan T (2019) Emergence of plasmid stability under non-selective conditions maintains antibiotic resistance. Nat Commun 10(1):1-13

35. Morlon J, Sherratt D, Lazdunski C (1988) Identification of functional regions of the colicinogenic plasmid ColA. Mol Gen Genet MGG 211(2):223-230
36. Song M, Kim HJ, Kim EY, Shin M, Lee HC, Hong Y, Rhee JH, Yoon H, Ryu S, Lim S, Choy HE (2004) ppGpp-dependent stationary phase induction of genes on Salmonella pathogenicity island 1. J Biol Chem 279(33):34183-34190

37. Zaharuddin L, Mokhtar NM, Nawawi KNM, Ali RAR (2019) A randomized double-blind placebo-controlled trial of probiotics in postsurgical colorectal cancer. BMC Gastroenterol 19(1):131

38. Österlund P, Ruotsalainen T, Korpela R, Saxelin M, Ollus A, Valta P, Kouri M, Elomaa I, Joensuu H (2007) Lactobacillus supplementation for diarrhoea related to chemotherapy of colorectal cancer: a randomised study. Br J Cancer 97(8):1028-1034

39. Percie du Sert N, Hurst V, Ahluwalia A, Alam S, Avey MT, Baker M, Browne WJ, Clark A, Cuthill IC, Dirnagl U, Emerson M, Garner P, Holgate ST, Howells DW, Karp NA, Lazic SE, Lidster K, MacCallum CJ, Macleod M, Pearl EJ, Petersen OH, Rawle F, Reynolds P, Rooney K, Sena ES, Silberberg SD, Steckler T, Würbel H (2020) The ARRIVE guidelines 2.0: updated guidelines for reporting animal research. J Cereb Blood Flow Metab 40(9):1769-1777

40. Min J-J, Nguyen VH, Kim H-J, Hong Y, Choy HE (2008) Quantitative bioluminescence imaging of tumor-targeting bacteria in living animals. Nat Protoc 3(4):629-636

Publisher's Note. Springer Nature remains neutral with regard to jurisdictional claims in published maps and institutional affiliations. 